\title{
Evolución de la planificación territorial en Perú 1920-2021: una aproximación histórica
}

Recepción: 17 de octubre de 2021 • Evaluación: 17 de diciembre de 2021 • Aprobación: 20 de enero de 2022

\begin{abstract}
Resumen: La planificación territorial en Perú es una política que ha recorrido un sinuoso proceso a lo largo de siete décadas de existencia sin que se haya consolidado como un instrumento orientador para el desarrollo del Perú. El objetivo de este estudio consistió en el análisis del proceso de planificación en el país a través del tiempo, prestando especial atención a los logros y las deficiencias que ha presentado en su recorrido histórico. La metodología utilizada en el estudio se basó en la revisión documental y normativa. Del análisis se desprende que el proceso de planificación en Perú se ha desarrollado en cuatro periodos importantes, denominados incipiente, apogeo, crisis y reinicio, cada uno de los cuales está intrínsecamente ligado a los acontecimientos políticos y socioeconómicos del país.
\end{abstract}

Palabras clave: planificación territorial, Perú, desarrollo, política.

Para citar: Rendón Cusi, S. F. (2022). Evolución de la planificación territorial en Perú 1920-2021: una aproximación histórica. Perspectiva Geográfica, 27(1), 32-50. https://doi. org/10.19053/01233769.13523 


\title{
Evolution of territorial planning in Peru 1920-2021: a historical approach
}

\begin{abstract}
Territorial planning in Peru is a political policy that has gone through a winding process throughout seven decades without having been consolidated as a guiding instrument for the development of Peru. The objective of this study was to analyze the planning process in the country over time, paying special attention to the achievements and deficiencies it has presented throughout its history. The methodology used in the study was based on documentary and normative review. The analysis shows that the planning process in Peru has developed in four important periods, called incipient, apogee, crisis and restart, each of which is intrinsically linked to the political and socioeconomic events of the country.
\end{abstract}

Keywords: territorial planning, Peru, development, politics

\section{Evolução do planejamento territorial no Peru 1920-2021: uma aproximação histórica}

\begin{abstract}
Resumo: O planejamento territorial no Peru é uma política que tem percorrido um processo sinuoso ao longo de sete décadas de existência sem que se tenha consolidado como um instrumento orientador para o desenvolvimento do Peru. O objetivo deste estudo foi a análise do processo de planeamento no país ao longo do tempo, prestando especial atenção às realizações e às deficiências que tem apresentado no seu percurso histórico. A metodologia utilizada no estudo baseou-se na revisão documental e regulamentar. Da análise se depreende que o processo de planejamento no Peru se tem desenvolvido em quatro períodos importantes, denominados incipiente, apogeu, crise e reinício, cada um dos quais está intrinsecamente ligado aos acontecimentos políticos e socioeconômicos do país.
\end{abstract}

Palavras-chave: planejamento territorial, Peru, desenvolvimento, politica. 


\section{Introducción}

La planificación territorial es un proceso integral e interdisciplinario que realiza acciones orientadas al desarrollo socioeconómico, sin embargo, en el Perú se ha visto limitada por los desaciertos que ha tenido a lo largo de su existencia. En este estudio buscamos definir las características del proceso peruano a través del análisis histórico de la planificación de acuerdo con el contexto en que se ha desarrollado. El periodo que abarca este estudio va desde la tercera década del siglo pasado hasta la actualidad (1920-2021) y ha sido dividido en cuatro etapas con sus respectivas características. Estos cien años han estado marcados por una serie de acontecimientos externos e internos que han sido determinantes para esta política estatal porque si bien la planificación es una negociación de interés colectivo entre públicos y privados, no siempre ha primado el consenso, lo que ha dificultado el proceso. La trayectoria de la planificación territorial en Perú evidencia una serie de problemas asociados tanto a la conceptualización como a la praxis, particularmente en las etapas de formulación, implementación y monitoreo. Consecuentemente, ningún proyecto de planificación territorial nacional ha podido concretarse y, por lo tanto, no puede decirse que se hayan podido cumplir los objetivos para los cuales se desarrollaron inicialmente.

La metodología de estudio consistió en la revisión y el análisis de la producción académica sobre esta temática, así como de los documentos oficiales elaborados por las entidades del Estado peruano. Del mismo modo, se ha revisado la información disponible en diferentes repositorios institucionales, archivos digitales y páginas web especializadas. Dentro del registro llevado a cabo, destacan los documentos institucionales de carácter informativo, jurídico y administrativo provenientes del sector gubernamental, fuentes oficiales entre las que destacan el Ministerio del Ambiente (MINAM), el Centro Nacional de Planeamiento Estratégico (CEPLAN) y el Instituto de Investigaciones de la Amazonía Peruana (IIAP). Para el análisis documental, se ha realizado una definición de etapas temporales, la clasificación de acontecimientos para cada una de ellas y el análisis narrativo de los sucesos, todo lo cual ha permitido caracterizar en su conjunto el proceso de la planificación territorial en Perú.

Esta periodización permite comprender en una perspectiva temporal la evolución de la planificación, desde la regional que surgió en la década de los treinta del siglo pasado hasta lo territorial y ambiental que rigen en la actualidad. En el primer periodo, denominado "incipiente", destaca el empleo de metodologías poco sofisticadas para la planificación sectorial y la planificación regional a través de cuencas hidrográficas. En el segundo periodo, denominado "apogeo", se realizó una intensa labor de planificación regional a partir de la creación del Instituto Nacional de Planificación (INP), entidad que tuvo un importante papel en la historia contemporánea del país, al mismo que tiempo que se formularon planes sectoriales con criterios y técnicas más rigurosos. El tercer periodo, denominado "de crisis", guarda relación con la desactivación del INP y el abandono de las experiencias acumuladas en el proceso. Finalmente, el cuarto periodo, designado como "reinicio", está marcado por el surgimiento de nuevos tipos de planificación, entre las que destacan la ambiental, la estratégica y la sectorial, bajo la rectoría del CEPLAN.

El trabajo está dividido en una introducción y cuatro secciones. La primera de ellas analiza los conceptos teóricos y las categorías usadas en los distintos tipos y modelos de planificación. La segunda sección esquematiza el proceso de la planificación y está dividida en cuatro subsecciones. La primera subsección se refiere al primer momento de la planificación territorial y considera los hechos previos a la consolidación de esta. En la segunda subsección se profundiza en los factores determinantes para la institucionalización de la planificación y el contexto que permitió su desarrollo. En la tercera subsección se explican las causas que condujeron a la crisis de la planificación y el retroceso en los avances logrados hasta ese momento por el 
proceso, que incluso llegaron a perderse. En la cuarta subsección se explica el reinicio de la planificación territorial con otros conceptos y perspectivas orientadas a la profundización de la planificación estratégica y la sectorial. Finalmente, se presentan las conclusiones.

\section{Planificación, un concepto dinámico}

La evolución de la planeación, tanto desde el punto de vista teórico como desde el práctico, ha incorporado una serie de cambios en su proceso. Esta trayectoria de carácter procesal ha experimentado cambios, pasando de la planeación económica a la planificación regional, de la planificación regional a la planificación estratégica y territorial y, finalmente, de la planificación estratégica y territorial a la planificación ambiental. A continuación, se explican los conceptos más importantes utilizados como marco teórico y práctico en la planificación regional, estratégica, territorial y ambiental, los mismos que se han puesto en marcha en los diferentes procesos desarrollados en Perú.

La planificación regional es tanto una disciplina como una metodología para planificar regionalmente a través de la organización de las fuerzas y los recursos regionales, orientados al desarrollo integral de la sociedad en el plano económico y social. Otros objetivos de la planificación regional han estado relacionados con la integración, la disminución de los desequilibrios productivos y demográficos, el control de la metropolización y el desarrollo de territorios específicos (Coraggio, 1981). Los ejemplos más concretos de planificación regional se desplegaron en Norteamérica y Europa, y los países latinoamericanos, entre ellos el Perú, replicaron estas experiencias. Entre los modelos de planificación regional que influyeron notablemente sobre los políticos y los planificadores del país andino podemos mencionar la Tennessee Valley Authority (TVA), que ya en 1933 planteó en Estados Unidos el desarrollo integrado de cuencas hidrográficas. Otros referentes han sido experiencias europeas como la política regional italiana, creada en 1950 a través de la Cassa per il Mezzogiorno, que tuvo como objetivo potenciar la industrialización, el incremento de la producción agrícola y la creación de infraestructura, como factores para el desarrollo socioeconómico. También podemos destacar las políticas del Town and Country Planning británico y el aménagement du territoire francés, que en 1955 se constituyó como una política gubernamental.

El concepto de planificación estratégica surgió en la década de los ochenta en la Escuela de Negocios de Harvard y, en primera instancia, fue aplicado a la empresa privada con el objetivo de alcanzar un alto grado de competitividad en el mercado y el éxito empresarial (Elizalde, 2003). En la actualidad esta herramienta se utiliza en la formulación de planes de desarrollo regional y local con criterios participativos que consideran las potencialidades y las debilidades del territorio, para lo cual se plantean lineamientos estratégicos para el desarrollo y la competitividad de las regiones y localidades. Este modelo de planificación es de carácter normativo, sistemático, estratégico y participativo, una estrategia que busca establecer escenarios futuros integrando diversas dimensiones de desarrollo, además de generar consensos sociales y espacios colaborativos entre los actores del territorio (Mata \& Quevedo, 1994).

La planificación territorial en el ámbito europeo se conoce como "ordenación del territorio" y en el Perú como "ordenamiento territorial". Según la Conferencia Europea de Ministros Responsables de la Ordenación del Territorio (CEMAT), la ordenación del territorio es "una disciplina científica, una técnica administrativa y una política concebida como un enfoque interdisciplinario y global; cuyo objetivo es un desarrollo equilibrado de las regiones y la organización física del espacio según un concepto rector" (CEMAT, 1983). Por consiguiente, la ordenación del territorio o planificación territorial se presenta como un modelo de carácter integral que articula las variables socioeconómicas, ambientales y territoriales en un determinado ámbito. 
Así mismo, Manuel Glave opina que "la planificación y el ordenamiento territorial tienen por finalidad complementar la planificación económica, social y ambiental con la dimensión territorial, racionalizar las intervenciones sobre el territorio y orientar su conservación y aprovechamiento sostenible" (Glave, 2012, p. 138). Por su parte, el geógrafo Horacio Capel (2016) indica que la planificación territorial no puede estar determinada solo por la virtud de los objetivos económicos a corto plazo, sino que también debe considerar aspectos sociales, culturales y ambientales, por lo cual es necesaria una aproximación al espacio y al objeto de la planificación. Por consiguiente, Antonio de Lisio (2014) sugiere que se debe edificar una combinación de objetivos sociales, económicos, políticos, ambientales y territoriales que recojan de manera esquemática los valores, los fundamentos y los medios de los ámbitos de intervención.

La planificación ambiental surgió en la década de los setenta, se consolidó en los ochenta y se intensificó después de la Eco 92 de Río. Para la Comunidad Europea, la planificación ambiental

[...] es una disciplina relativamente nueva orientada a coordinar la planificación urbana/regional con las cuestiones ambientales. La expresión más común de la planificación ambiental es la evaluación del impacto ambiental de los proyectos y programas sobre el uso del suelo, el desarrollo económico, el transporte, el desarrollo inmobiliario, el aire, el ruido, el agua, los humedales, las especies en peligro de extinción y los hábitats, los ecosistemas, las zonas inundables, zonas costeras, aspectos visuales, etc. (Pemán, 2011, p. 447).

El principal objetivo de la planificación ambiental es la toma de decisiones sobre el componente espacial y ecológico y la problemática por resolver es la interacción existente entre la sociedad y la naturaleza, lo que requiere una intervención sobre el uso sostenible de los recursos no renovables, considerando las limitaciones y potencialidades del medio natural. Los planteamientos teóricos sostienen que la planificación ambiental se hace efectiva con un ordenamiento ambiental del territorio orientado hacia un uso sostenible y un manejo adecuado, ya que regula las actividades económicas que afectan o impactan al medio ambiente, pero, si bien se esboza como una alternativa conducente al desarrollo, es un planteamiento que carece de realismo en el complejo escenario económico que presenta el mundo.

Una de las mayores preocupaciones de la planificación ambiental es el impacto de las actividades económicas, situación que ha ayudado a la generación de una frondosa normativa medioambiental y a la protección de la biodiversidad. Glave sugiere que es necesario el establecimiento de las condiciones de uso y de ocupación del territorio de acuerdo con las características ecológicas de este, teniendo en cuenta la fragilidad, la vulnerabilidad y el endemismo de los ecosistemas y las especies (Glave, 2012, p. 25). Desde la perspectiva de los macrosistemas, Guimaraes sostiene que los humanos constituyen el centro y la razón del desarrollo, pero reconoce la urgencia de un nuevo estilo que sea "ambientalmente, socialmente, culturalmente, políticamente y éticamente sostenible" (Guimaraes, 2001 p. 51). Sin embargo, el corpus normativo aprobado por el Estado peruano para la gestión ambiental se caracteriza por ser disperso, indefinido y ambiguo. Según Rendón (2019), en la legislación peruana hay una diversidad de conceptos como ordenamiento ambiental, ordenamiento ambiental territorial, ordenamiento territorial ambiental y planificación ambiental, que son utilizados indistintamente y vinculados a un mismo problema.

\section{Proceso de la planeación en Perú}

El proceso de planeación en Perú ha recorrido una trayectoria sinuosa. En la Figura 1 se han resumido los cuatro períodos de su evolución temporal, que abarca desde la tercera década del siglo xx hasta la actualidad. Estos han sido denominados como 
incipiente, apogeo, crisis y reinicio y están caracteri- y la práctica de la planificación llevada a cabo, todos zados por la intervención de distintos tipos y modelos los modelos están intrínsecamente relacionados con de planificación: la planificación regional, la estratégica, la territorial y la ambiental. Sin embargo, como sugiere Castillo (2021), cualquiera que fuese la teoría la "planificación del desarrollo", entendida como un proceso racionalizador en la toma de las decisiones para el avance del país.

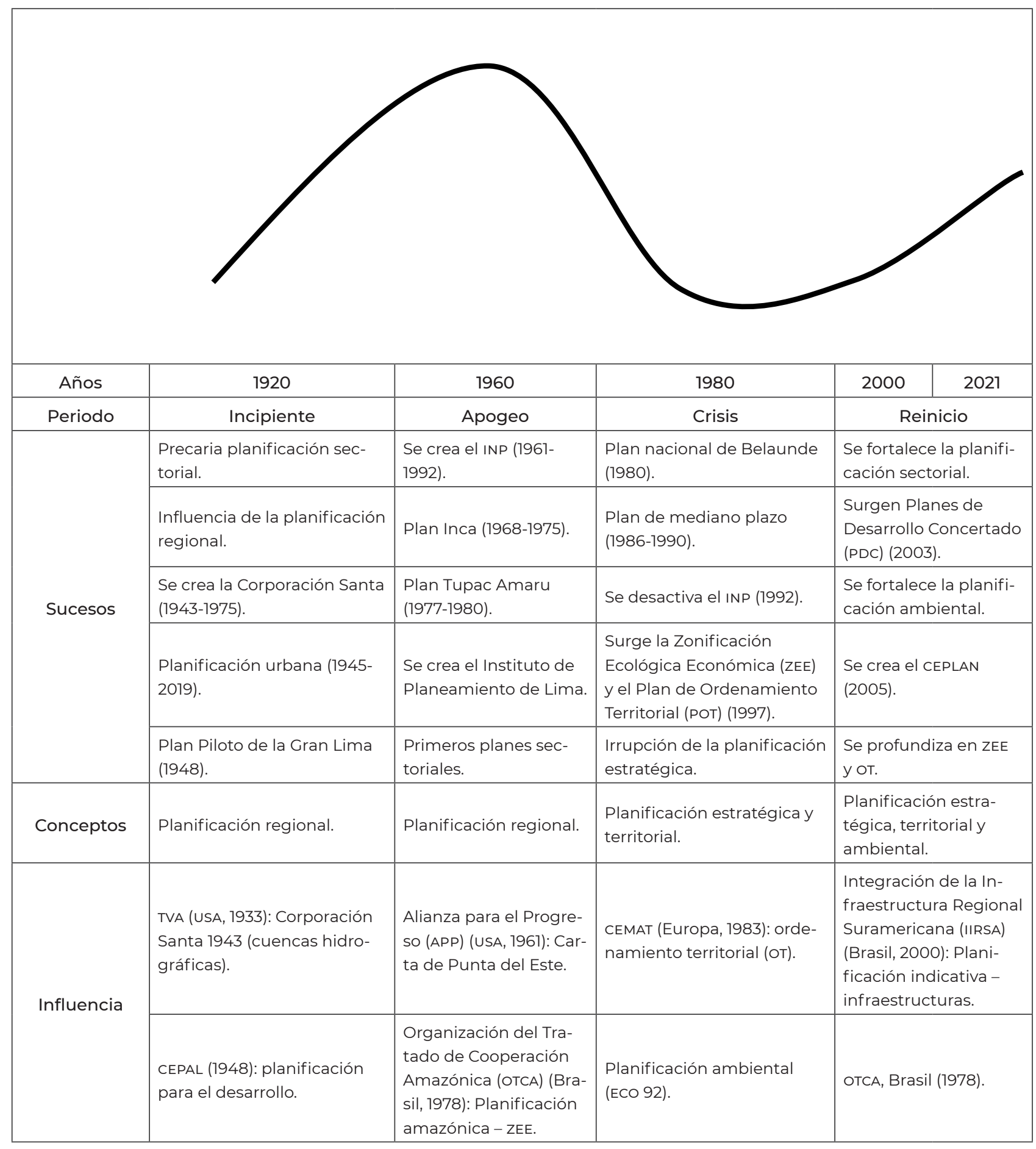

FIGURA 1. Períodos de la planificación en Perú

FUENTE: elaboración propia. 


\subsection{Primer periodo: planificación incipiente (1920-1960)}

Los primeros atisbos de planificación económica y sectorial con criterios técnicos surgieron en la tercera década del siglo xx, donde destaca la planificación vial para la construcción de carreteras y la infraestructura para la irrigación. Estas acciones fueron la cara principal del régimen del oncenio de Leguía (1919-1930), ya que este régimen vendía la imagen de un Estado planificador y preocupado no solo por un horizonte de corto plazo, sino de larga proyección (Zegarra, 2020). De acuerdo con Humberto Leceta (2012), el aprista Víctor Raúl Haya de la Torre también sugirió la necesidad de adoptar un plan reorganizador basado en la investigación científica de la realidad socioeconómica del país y en la misma línea opinaba el fundador del Partido Comunista del Perú, José Carlos Mariátegui, quien enfatizó en la función planificadora del Estado. Ninguna de las dos propuestas tuvo preponderancia en el escenario peruano de la época, sino que acabaron siendo complementarias a las políticas de planificación regional del Perú.

En las postrimerías del gobierno de Leguía, la situación económica del país decreció debido a la depresión de Estados Unidos de 1929, que se extendió por una década hasta que en el país norteamericano se emprendieron desafiantes políticas económicas conocidas como el New Deal (Bielza de Ory, 2002). Paralelamente, el Perú afianzó el desarrollo de la planificación regional y en la década de 1940 empleó el modelo de desarrollo integrado de las cuencas hidrográficas, que había sido implementado previamente por la Tennessee Valley Authority en Estados Unidos. Esta experiencia se replicó en 1943 en el proyecto de la Corporación Peruana del Santa, que buscó promover el desarrollo regional integral del norte del Perú por medio de inversiones en una obra de hidroeléctrica en el cañón del Pato, en una industria siderúrgica de Chimbote y en infraestructura básica. Asimismo, en este periodo se expandió la burocracia estatal y con ello la necesidad de planificar sus acciones, por lo que la planificación regional se constituyó como un marco general para la formulación de planes sectoriales en educación, salud y vivienda, entre otros, acciones que se fortalecieron en función del crecimiento del aparato estatal y adquirieron protagonismo en los años posteriores (Lira, 2006).

En 1948 surgió la Comisión Económica para América Latina y el Caribe (CEPAL) con el objetivo promover el crecimiento económico equitativo y la asignación de recursos financieros para contribuir al desarrollo de los países de América Latina y el Caribe. Sin embargo, sus designios no necesariamente tuvieron el éxito que esperaba esta institución multilateral. Influenciada por las experiencias italiana y francesa, así como por el pensamiento keynesiano, en el periodo de 1948 a 1960 la CEPAL promovió la integración regional y la industrialización por sustitución de importaciones (ISI) con el objetivo de impulsar el desarrollo productivo dirigido por el Estado. Siguiendo estas premisas desarrollistas, tal como indica Fitzgerald (1981), hacia mediados del siglo xx la planificación regional se había integrado en la retórica de los tecnócratas progresistas, quienes a su vez influyeron en el aparato estatal que creó juntas departamentales para planificar el desarrollo de estas jurisdicciones. En definitiva, en este periodo hubo avances significativos en la planificación regional y las estrategias de desarrollo se manifestaron a través de la construcción de equipamientos e infraestructuras de transporte para la integración nacional.

\subsection{Segundo periodo: auge de la planificación (1960-1980)}

Hacia la década de los sesenta la situación socioeconómica peruana se había complejizado aún más debido a las demandas de una reforma agraria por parte de los campesinos, particularmente de la zona andina y de la ceja de selva, que estaban influenciados 
por la Revolución cubana. Además, como señala Fitzgerald (1981), en Perú existían fenómenos estructurales subyacentes debido al proceso político de industrialización por sustitución de importaciones y la disminución de la inversión privada. Esta situación ha sido confirmada por la CEPAL, que asevera que la economía del Perú en la década de los sesenta era de "inserción débil y vulnerable" debido a la carencia de infraestructura productiva y competitiva. En consecuencia, se requería una intervención inmediata en los diferentes estamentos del aparato estatal mediante la praxis de la planeación, lo que significó un ejercicio de enorme desafío.

La intervención de Estados Unidos fue decisiva para las políticas de planificación latinoamericanas, ya que, en virtud de distintos acuerdos, los países firmantes realizaron reformas y formularon planes. La reunión de Punta del Este (Uruguay) en 1961, impulsada por Estados Unidos a través de la plataforma política Alianza para el Progreso, se realizó bajo la premisa de mejorar la vida de todos los habitantes del continente. Con este objetivo principal en mente, se buscó consolidar gobiernos democráticos, eliminar el analfabetismo, aplicar reformas agrarias y reducir las brechas socioeconómicas a través de la planificación regional. Con la finalidad de concretar estas metas, Estados Unidos ofreció ayuda económica, técnica, política y social a los países firmantes, entre ellos, Perú. Esta política del presidente Kennedy fue una respuesta para contrarrestar la influencia de la Revolución cubana, que había incidido en el avance del socialismo en la región, escenario que alteraba a la hegemonía estadounidense en el continente.

En virtud del acuerdo, el 19 de octubre de 1962 se aprobó la Ley n ${ }^{\circ} 14.220$ del Sistema Nacional de Planificación para el Desarrollo Económico y Social del Perú, integrada por el organismo técnico central del Instituto Nacional de Planificación (INP), el Concejo Nacional de Desarrollo Económico y Social (CNDES), las Oficinas Sectoriales de Planificación (OSP) y las Oficinas Regionales de Planificación (ORP). Con esta estructura institucional, el objetivo principal fue el de mejorar las condiciones del ciudadano peruano a través de la ejecución de planes sistemáticos para el desarrollo económico y social, razón por la cual desde su constitución institucional en 1962 y hasta 1979 se intensificó la formulación e implementación de diversos planes para el desarrollo nacional.

El recién creado Instituto Nacional de Planificación presentó en 1964 el primer Plan de Desarrollo Económico y Social de carácter sectorial para el período comprendido entre 1967 y 1970, que, si bien se fundamentaba en las premisas de la planificación regional, no llegó a ejecutarse (Fitzgerald, 1981). En este contexto, al Perú le interesaba contar con planes en el marco de la política de la Alianza para el Progreso de Estados Unidos y así beneficiarse de los servicios financieros acordados en la Carta de Punta del Este, es decir, la formulación de planes era una preocupación política derivada de un acuerdo externo que se debía cumplir.

A pesar de estas deficiencias, hubo aciertos como la creación de la Oficina Nacional de Evaluación de Recursos Naturales (ONERN) en 1962, que realizó los primeros inventarios de recursos naturales, estudios multidisciplinarios y el desarrollo de la cartografía por temáticas como tipo de suelos y capacidad de uso mayor de tierras, entre otras. Por otro lado, con objetivo de descentralizar el país, en 1964 se planteó la creación de corporaciones de desarrollo departamental con el objetivo de incorporar la dimensión espacial en los planes de desarrollo. Por lo tanto, con el fin de hacer más efectiva la práctica de la planeación, se regionalizó el Perú en corporaciones con el presupuesto del Fondo Nacional para el Desarrollo Económico, cuyo sistema de financiamiento jugó un papel preponderante en algunos departamentos como Arequipa, Piura y San Martín (Hilhorst, 1979). El planteamiento de las propuestas se basó en los fundamentos de la planificación regional de origen europeo, particularmente en el modelo francés de aménagement du territoire, que los planificadores regionales peruanos 
reprodujeron en los procesos para la descentralización, tanto en los polos de crecimiento como en los centros de desarrollo.

Las crisis externas e internas fueron determinantes para el éxito o el fracaso de la planificación. Después del golpe de Estado del general Juan Velasco Alvarado al gobierno de Fernando Belaunde Terry en octubre de 1968, el régimen militar fortaleció una planificación planteada desde el crecimiento económico y el progreso social, con nuevas políticas de desarrollo y el uso de conceptos como la redistribución de la riqueza, de la tierra y del poder. A través de la reforma agraria se realizaron profundas transformaciones en la ocupación del territorio, ya que la posesión de las tierras estaba en manos de una minoría latifundista y sus habitantes estaban sometidos a condiciones infrahumanas. Otoniel Velasco (2013) afirma que el régimen militar de Juan Velasco Alvarado aspiraba a que la revolución lograse la construcción de una democracia social con participación plena de los ciudadanos.

La década de los setenta fue presidida por el Gobierno Revolucionario de las Fuerzas Armadas. Durante el primer periodo, encabezado por Juan Velasco Alvarado (1968-1975), se promovió el Plan Inca y en el segundo periodo, presidido por Francisco Morales Bermúdez (1975-1980), se llevó a cabo el Plan Túpac Amaru (1977-1980). Durante el gobierno de Velasco hubo un clima planificador favorecido por la profesionalización del recurso humano en planificación regional y por el avance de los conocimientos técnicos para resolver problemas regionales. Por lo tanto, tal como afirma Sánchez (2002), se afianzó una "verdadera planificación estatal del desarrollo socioeconómico, una política exterior en defensa del territorio y de los intereses soberanos del Perú" (p. 245). Aunque la retórica era bien intencionada, la práctica se perdió en el "voluntarismo utópico, el reduccionismo economicista y el formalismo" (Lira, 2006, p. 11). A pesar de las deficiencias en el sistema de planificación, el Gobierno prosiguió con el proceso de la planeación, lo que evidencia la existencia de voluntad política para hacer de la planificación un instrumento fundamental para lograr un elevado grado de coordinación y coherencia entre la macroeconomía, las políticas sectoriales y la política social.

En 1971, el gobierno militar de Velasco aprobó el Decreto Ley 18.742, el cual precisaba el papel del Instituto Nacional de Planificación como organismo proveedor, coordinador y evaluador del programa nacional de cooperación técnica. En consecuencia, el Instituto Nacional de Planificación se encargó de implementar políticas de desarrollo a través de sus oficinas de planificación sectorial en ministerios y regiones, que en un principio eran cuatro y que a partir de 1972 se incrementaron a nueve. Así mismo, entre 1971 y 1975 se implementó un Plan Nacional de Desarrollo subdividido en dos periodos de ejecución, el primero entre 1971 y 1972 y el segundo de 1973 a 1974. Este se aplicó sobre la base de planes sectoriales (anteproyectos) y las previsiones económicas producidas por el Instituto Nacional de Planificación, pero sin la participación del sector privado (Fitzgerald, 1981). En consecuencia, el Estado se convirtió en el principal inversor y empleador y la planificación fue la herramienta principal para la aplicación de los programas, aunque en un escenario de rígida estructura social caracterizada por la concentración del poder económico y político por parte de una minoría.

En definitiva, la planificación se erigió como un instrumento de transformación estructural orientado a beneficiar a vastos sectores de la población ajenos a la distribución de la riqueza que recaía en sectores privilegiados. Para alcanzar estos objetivos se incorporaron los criterios de la planificación regional o espacial basados en la división del país en cuatro grandes regiones (norte, centro, sur y oriente), y para su efecto se formularon planes quinquenales con estrategias de mediano y corto plazo. Esta apuesta estatal produjo un efecto positivo, ya que por primera vez en la historia del Perú se implementaba una política de desarrollo ordenada y planificada, hecho que coincide con el apogeo alcanzado por la planificación regional en 
Perú debido a la decisión política del gobierno militar. Es así como podemos señalar que parte de la década de los setenta del siglo xx fue la más importante para la planificación debido a que en estos años se implementaron transformaciones y reformas en diversos aspectos como el agrario, el educativo y el de la administración pública, y que, en definitiva, las estrategias de desarrollo rural llevaron a la consolidación de la práctica de la planificación y sus alcances.

El Instituto Nacional de Planificación (INP) tuvo un papel protagónico en la formulación de las políticas de desarrollo, ya que entre los años 1973 y 1974 las oficinas regionales de planificación departamental adquirieron un nuevo enfoque. Sin embargo, los problemas derivados de los cambios en el Instituto acabaron ahogando el proceso a raíz que "las oficinas regionales de planificación regresaban una y otra vez a la elaboración de diagnósticos y a la formulación de estrategias y objetivos, pero las ausencias de contrapartidas en las regiones convirtieron su trabajo en un esfuerzo sin respuesta" (Hilhorst, 1979, p. 39). Las políticas de descentralización no funcionaron adecuadamente a causa del centralismo de la capital nacional y la dependencia del país de las decisiones externas, lo que se vio incrementado por la deuda externa y la crisis económica mundial. En ese contexto, los objetivos de desarrollo regional no llegaron a concretarse, y, dado que los resultados económicos acabaron siendo adversos para el régimen de Velasco, la planificación acabó sufriendo un revés debido a la crisis del petróleo.

En 1975 la situación empeoró con la caída de Velasco, ya que la planeación se vio seriamente afectada por la crisis económica. Este escenario fue aprovechado por el siguiente régimen, también militar, y Morales Bermúdez acometió una serie de contrarreformas. A pesar de los cambios acaecidos en este gobierno, emplearon la teoría y la práctica de la planificación regional para formular el Plan Nacional de Desarrollo (1975-1978), que fue aprobado con el Decreto Supremo $n^{\circ}$ 009-75 el 2 de agosto de 1975. El objetivo de este plan era constituirse como marco orientador para la formulación e implementación de planes bienales, llamados también "planes a corto plazo", formulados para los periodos de 1975-1976 y 1977-1978, que fueron elaborados en forma sectorial y departamental para luego ser integrados a un "plan global" (CEPAL, Instituto Latinoamericano y del Caribe de Planificación Económica y Social [ILPES], 1978, p. 107) con el objetivo de "estabilizar y reactivar la economía" (Fitzgerald, 1981). En este periodo se intentó fortalecer la planificación a través del Plan Túpac Amaru (19771980), el cual propuso lineamientos para superar la crisis económica existente en el país desde 1975. En definitiva, pese que se había emprendido una contrarreforma, se empleaba aún el discurso revolucionario del gobierno de las Fuerzas Armadas.

En un principio, algunas ideas del primer gobierno militar mantuvieron su vigencia y se aspiraba a consolidar los objetivos en el aspecto social con el fin de corregir los problemas medulares que aquejaban a la sociedad peruana. A pesar de ello, con el devenir de los meses se comenzó a desandar el camino recorrido por Velasco, se desactivó el Sistema Nacional de Apoyo a la Movilización Social (SINAmos) y la Confederación Nacional Agraria (GNA) y se crearon en su lugar los Organismos de Desarrollo (ORDEs), orientados a impulsar la construcción de grandes infraestructuras para la producción de energía y hierro. El Plan Túpac Amaru tuvo como finalidad "consolidar el proceso revolucionario, evitando que derive hacia el estatismo comunista y que, por reacción, retroceda a formas ya superadas del capitalismo pre-revolucionario; completar y reajustar las reformas estructurales" (Leceta, 2012, p. 65). Los planes de corto plazo formulados buscaron superar los problemas precedentes, pero igualmente fueron cuestionados porque seguían siendo rígidos, ortodoxos y basados "en planes economicistas e incompletos" (Gallardo, 2013, p. 31), además de burocráticos y utópicos, ajenos a las aspiraciones de integralidad, es decir, tampoco lograron superar las expectativas que se tenían en la planificación. 
Según Leyton y López (2005), poco a poco la planificación fue quedando deslegitimada, ya que el modelo suponía una subordinación de las organizaciones sociales y económicas al Estado, en particular, el Sistema Nacional de Apoyo a la Movilización Social (SINAMOS). Esto generó un conflicto entre dos entidades de carácter diferente, el SINAMOs, que era eminentemente político, y el Instituto Nacional de Planificación (INP), una instancia tecnocrática del Estado. Sergio Galilea (1979) agrega que la planificación de la época tuvo un contenido sectorial y que al crearse oficinas de planificación en diferentes ministerios se generaron disputas por los recursos necesarios para financiar los proyectos, que luego trascendieron y evolucionaron hacia perspectivas territoriales y multisectoriales.

El Plan Túpac Amaru se diseñó considerando tanto los fracasos como los éxitos obtenidos por los planes previos. Entre los criterios establecidos encontramos el perfeccionamiento y la promoción concertada de los sectores económicos, así como la necesidad de acometer "reajustes en las reformas estructurales para luego institucionalizarlas por medio de una Constitución" (Lajo, 1980, p. 203). En medio de la crisis y el descontento social, el gobierno militar convocó una Constituyente que instituyó una nueva Carta Magna en 1979. Según Sánchez (2003), esta Constitución se caracterizó por el "constitucionalismo social" y la función relevante del Estado en la asignación de recursos con ciertas dosis de intervención pública. Esta nueva Constitución reconocía la planificación y los planes de desarrollo a escala nacional, regional y local, afirmando que "el Estado formula la política económica y social mediante planes de desarrollo que regulan la actividad de los demás sectores. La planificación una vez concertada es de cumplimiento obligatorio" (Constitución Política del Perú, 1979, art. 111). Sin embargo, los efectos tardíos de la crisis del petróleo generaron un panorama de incertidumbre para la planificación estatal, que ya venía siendo cuestionada debido a los escasos resultados alcanzados después de una intensa década de planificación para el desarrollo. En resumen, este periodo representa los "años dorados" de la planificación nacional por el compromiso político que tuvieron los diferentes gobiernos, por su institucionalización y por la apuesta de formulación y aplicación de planes; sin embargo, solo tuvo éxitos relativos y resultados disímiles en las regiones, lo que derivó en que la planificación empezó a percibirse como una política negativa.

\subsection{Tercer periodo: crisis de la planificación (1980-2000)}

Este periodo se enmarca en el contexto del surgimiento de la planificación estratégica y territorial, así como de las propuestas neoliberales y, por consiguiente, en el descrédito de la planificación regional. Con la promulgación de la nueva Constitución en julio de 1979, se convocó a elecciones y fue elegido para un segundo periodo presidencial Fernando Belaunde. En su gobierno se produjeron una serie de descoordinaciones que provocaron que se perdiese

[...] una gran parte del impulso adquirido, revirtiendo el proceso, mientras que las relaciones entre las principales entidades de planificación y las instituciones financieras del Estado se hicieron cada vez más conflictivas, agudizando rivalidades entre estas entidades sobre la formulación y control de la política económica (Ponce, 2014, p. 405).

En este periodo se volvió a la elaboración de planes de desarrollo basados en las proyecciones macroeconómicas de la economía peruana sin tomar en cuenta los antecedentes y algunos de ellos incluso llegaron a contemplar un financiamiento externo en un contexto desfavorable para el país. Según Fitzgerald (1981), esta situación evidencia que "la política, la economía y la planificación peruana no se desarrollaron articuladamente" (p. 339), sino que hubo una orientación hacia una política tradicional y hacia una planificación limitada al sector público.

Belaunde no era partidariode una planificación económica y social, sino que promovía una planificación 
de carácter físico o territorial (Velasco, 2013), por ejemplo, relacionada con la planificación urbana. Contrariamente al posicionamiento del presidente, en virtud del artículo 111 de la Constitución de 1979, en junio de 1981 se promulgó el Decreto Ley n 177 , denominado "Ley de organización y funciones del sistema nacional de planificación". Si bien esta norma incluía planteamientos ambiciosos y reestructuraciones en el sistema, la mayoría de las iniciativas fracasaron por falta de voluntad política del Ejecutivo y debido a la violencia promovida por el movimiento Sendero Luminoso. Otro factor que afectó a la planificación en esta década fueron los efectos tardíos de la crisis económica de la década de los setenta, que en Perú se manifestó más tempranamente que en el resto de los países latinoamericanos (Portocarrero, 1989) y se extendió hasta los primeros años de la década de los ochenta. Entre 1983 y 1985, el Gobierno intentó continuar débilmente con la planificación e incluso propuso su descentralización, pero ninguna de las propuestas prosperó a pesar de los esfuerzos gubernamentales, ya que si bien Belaunde logró estabilizar la economía peruana impulsando las exportaciones e incrementando la inversión público-privada a través de la apertura económica y de una menor intervención estatal, la crisis económica se profundizó y se vieron afectadas todas las políticas públicas, escenario que acabó siendo decisivo para el futuro de la planificación regional en Perú.

Con el ascenso al gobierno de Alan García (19851990), la situación socioeconómica del país se agravó debido a las políticas populistas emprendidas, como la restricción del pago de la deuda externa, la limitación de las importaciones y el apoyo crediticio sin intereses a la agricultura, medidas que buscaban cosechar éxitos a corto plazo, pero que a la larga generaron un elevado gasto público para el país (Portocarrero, 1989). Javier Tantalean, jefe del Instituto Nacional de Planificación, hizo los últimos esfuerzos de la institución presentando un Plan Nacional de Desarrollo para el período 1986-1990, documento elaborado por técnicos y burócratas formados en periodos anteriores al gobierno de García, que pretendió implementar una "mayor planificación estatal para la inversión selectiva de los escasos recursos que se disponía” (Reyna, 2000, p. 86). Sin embargo, a la par que decaía el crecimiento económico lo hacía el optimismo, ya que la situación económica del país se vio agravada por la hiperinflación y dio paso a una crisis financiera. Según la CEPAL (1986), esta situación se debió a que no hubo una correspondencia entre la estructura productiva y los patrones de consumo en el país, lo que generó un incremento del gasto en la importación de bienes.

En este contexto, los últimos atisbos de la planificación regional fueron la realización de tareas de diagnóstico y proyecciones globales, así como la planeación para sectores y proyectos específicos, pero la situación de conflicto interno y la corrupción del gobierno aprista imposibilitó avanzar en la concreción de las propuestas. Los resultados de la planificación en este periodo no fueron significativos, puesto que el manejo de la coyuntura y la emergencia continuaron en los ámbitos de la política económica. En 1990 el gobierno de García promulgó el Decreto Ley no 593 que incorporaba un mayor número de integrantes al Sistema Nacional de Planificación - en su mayoría, del Partido Aprista Peruano-, lo cual, en lugar de fortalecer la institución, provocó su mayor desprestigio. Además, se generalizó la idea de su incompetencia debido a que no ofrecía resultados positivos en contraposición al mercado, que sí podía resolver los problemas. En este contexto el mercado adquirió preponderancia por sobre el Estado, lo que provocó que se plantease la reducción de las competencias estatales y la disminución de sus capacidades, contraviniendo los planteamientos iniciales de la planificación regional y, consecuentemente, aumentando la preponderancia de la planificación estratégica.

Para la década de los noventa la planificación prácticamente estaba desacreditada y con la elección de Fujimori, un outsider de la política peruana hasta 
ese momento, aumentó la incertidumbre, ya que no había claridad sobre qué ocurriría en el país. Fujimori instauró un gobierno corrupto y autoritario que aplicó drásticas medidas neoliberales mediante un programa de ajuste estructural conocido como el "fujishock". Permitió la liberalización de la economía peruana y realizó extensas privatizaciones de las empresas públicas (Minero Perú, Entur Perú, AeroPerú y Entel Perú, entre otras). Por lo tanto, las políticas ligadas a la planificación dejaron de tener importancia, ya que se tildaban de burocráticas, ineficaces e inservibles para los complejos procesos de desarrollo. El gobierno apelaba al pragmatismo y al populismo y desarrolló una forma de resolución de los problemas públicos que resultó siendo más burocrática y totalmente antagónica a la planificación. En 1992 se aprobó el Decreto Ley n ${ }^{\circ} 25.548$ mediante el cual se disolvió el Instituto Nacional de Planificación (INP) y se creó en su lugar el Consejo Nacional de Planificación, que, conformado por ministros, no llegó a desarrollar ningún plan (Moschella, 2012). Las políticas aplicadas por Fujimori obedecieron al decálogo de reformas estructurales promovidas por el Consenso de Washington, que fueron empleadas en toda América Latina.

El gobierno de Fujimori, que se extendió durante toda la década de los noventa, representa no solo la disolución del Instituto Nacional de Planificación y su dispersión en diferentes esferas del Estado, sino también la destrucción y la desaparición del legado de treinta años de experiencia acumulada. Posteriormente, a través de la Ley $n^{\circ}$ 25.490, se creó el Ministerio de la Presidencia que, en lugar del Instituto Nacional de Planificación, dispuso de instituciones descentralizadas dependientes del mencionado ministerio; esta forma de gestión fue un instrumento de corrupción gubernamental. En esta década también irrumpieron los debates sobre el medio ambiente. Destaca la realización de la ECo 92 de Río, de la cual surgió con fuerza el concepto de desarrollo sostenible enfocado a la ordenación del territorio y la erradicación de la pobreza (Bielza de Ory \& Bohl, 2012).
Estas propuestas chocaron con el espíritu de la nueva Constitución Política del Perú de 1993, que optaba por un Estado liberal regulado por la actividad privada (Sánchez, 2003), en particular con las relacionadas con el aspecto económico porque el modelo se sustentaba en una economía primaria exportadora que los planes racionalizaban.

Con respecto a la planificación, esta fue mencionada tibiamente en el artículo 195, que planteaba que "los gobiernos regionales promueven el desarrollo y la economía regional, fomentan las inversiones, actividades y servicios públicos de su responsabilidad, en armonía con las políticas y planes nacionales y locales de desarrollo" (Constitución Política del Perú, 1993). Esta relación no se dio entre los niveles de gobierno; al contrario, tanto las gestiones regionales como locales desarrollaron sus políticas de desarrollo y sus planes de forma desarticulada. En este contexto surgieron propuestas de planes medioambientales y la Zonificación Ecológica Económica (ZEE) como base para el Plan de Ordenamiento Territorial (POT), la mayoría de los cuales estuvieron orientados a fortalecer planes sectoriales y la planificación urbana, ambiental y estratégica.

Se desconocieron completamente los avances de la planificación regional, cuyos sistemas entraron en una etapa de desarme, abandono sistemático y desmantelamiento de todo lo desarrollado en esta materia. Durante esta década la mayoría de las intervenciones se realizaron sin que existiese un sistema de planificación institucionalizada, sino de forma sectorial y controlada por el Poder Ejecutivo a través del Ministerio de la Presidencia. Así, el proceso no pudo ser fiscalizado para determinar la eficiencia o eficacia del empleo de los recursos públicos provenientes de las privatizaciones (Chiarella \& Yakabi, 2016). A partir de la mitad de la década los noventa se consolidó la planificación sectorial y la planificación estratégica que, originada en el ámbito empresarial norteamericano, se basaba en la premisa de la predictibilidad desarrollada a través de los modelos Harvard, Ansoff, Porter y el modelo de consultorías. Empleando los planteamientos del geógrafo 
Vicente Bielza de Ory (2012), el Perú ha mirado excesivamente al exterior para sus procesos de planificación y sus criterios de aplicación, cuando la realidad del país y sus características difieren de otros contextos.

\subsection{Cuarto periodo: reinicio de la planificación (2000-2021)}

En la primera década del siglo XxI hubo importantes avances en cuanto a la promulgación de normativas relacionadas tanto con la planificación estratégica como con la territorial y con la ambiental, razón por la que a este periodo temporal se ha denominado como un "reinicio" de la planificación. Después de la dictadura fujimorista el país retornó a la democracia, se recuperaron las instituciones y durante el breve gobierno de transición encabezado por Valentín Paniagua (2000-2001) se realizaron algunos avances relacionados con la política de planificación territorial, donde destaca, en el año 2001, la creación de la Comisión Nacional para el Ordenamiento Territorial Ambiental a través del Decreto Supremo nº 045.

En el siguiente periodo gubernamental, presidido por Alejandro Toledo (2001-2006), se recuperó la economía y el país experimentó un crecimiento debido al incremento de la inversión en actividades extractivas. En el año 2002 se aprobó el Acuerdo Nacional, en el que la planificación se consideró como la quinta política estatal y el Estado se comprometió a impulsar el planeamiento estratégico para alcanzar los objetivos nacionales de desarrollo, crecimiento e integración adecuados a la economía global. Esta decisión política contribuyó al fortalecimiento del planeamiento estratégico, por lo que el 6 de mayo de 2005, mediante la promulgación de la Ley n ${ }^{\circ}$ 28.522, se creó el Sistema Nacional de Planeamiento Estratégico (SINAPLAN) y el Centro Nacional de Planeamiento Estratégico (CEPLAN). A pesar de la existencia de una voluntad política y de una reglamentación correspondiente a la ley, la implementación del plan tardó en operativizarse por cuestiones burocráticas del sistema estatal y por los limitados recursos económicos destinados a este fin. Asimismo, los criterios para la formulación, la implementación, el seguimiento y la evaluación del proceso no fueron claros, por lo que, como señala Marc Dourojeanni (2016), la formulación acabó obedeciendo a un formalismo y los planes no se implementaron adecuadamente.

El SINAPLAN se caracteriza por ser un conjunto articulado e integrado de órganos, subsistemas y relaciones funcionales estatales cuya finalidad es la coordinación y la viabilización del proceso de planeamiento estratégico nacional, con el objetivo de promover y orientar el desarrollo armónico y sostenible del país, todo lo cual está moldeado por el contexto social (Servillo \& Van Den Broeck, 2012). El sistema está conformado por el Poder Ejecutivo, integrado por los ministerios, el Poder Legislativo, el Poder Judicial, organismos constitucionales autónomos (OCA), los gobiernos subnacionales (regionales y locales) y el Foro de Acuerdo Nacional, integrado por partidos políticos y organizaciones de la sociedad civil. Asimismo, como se observa en la Figura 2, en Perú los niveles de planeamiento estratégico están articulados en forma jerárquica.

En la segunda década del presente siglo (20112021), la planificación sectorial impulsada por los ministerios se ha impuesto frente a los demás enfoques de la planificación (territorial, estratégica y ambiental). Si bien en los primeros años del presente siglo se emprendieron planes estratégicos de desarrollo concertado, estas fueron experiencias de carácter no vinculante y completamente desligadas de los diferentes niveles de gobierno. Según Leyton y López (2005), las experiencias locales de planificación estratégica y concertada fueron importantes aportes para la articulación entre el nivel local y el regional, sin embargo, las relaciones estaban subordinadas a aspectos como el grado de desarrollo de los municipios, el tamaño de las jurisdicciones, el tamaño de la población y la capacidad institucional de los gobiernos locales para proponer iniciativas a los gobiernos regionales y para que estas fuesen viabilizadas adecuadamente, lo que dificultó su avance y realización. 


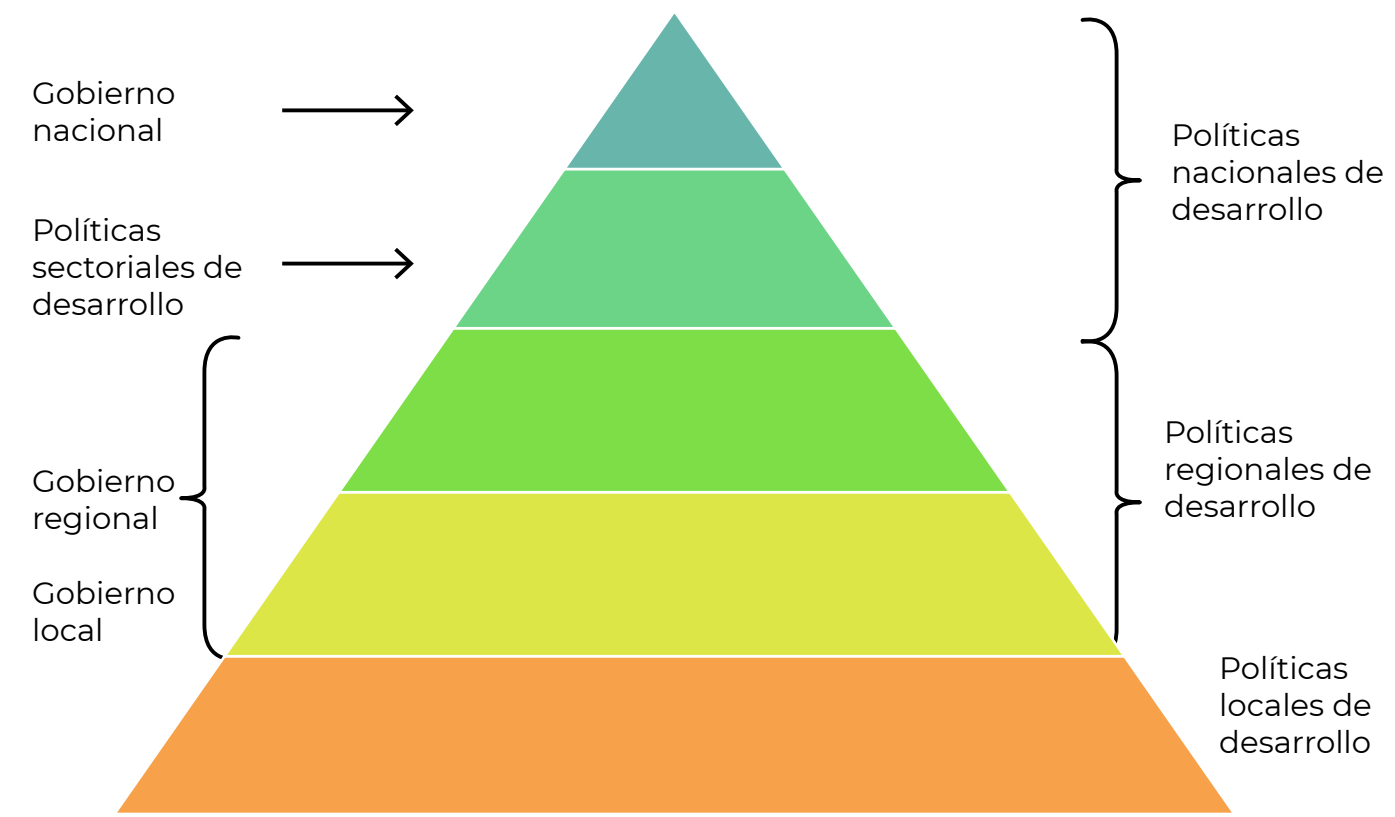

FIGURA 2. Niveles de planeamiento estratégico en Perú

FUENTE: CEPLAN (2015).

$\mathrm{Al}$ régimen de Humala se le conoció como el gobierno de "la gran transformación y la hoja de ruta" (2011-2016), sin embargo, su administración se caracterizó por una serie de contradicciones en torno a las políticas de planificación. En el marco de la planificación estratégica, en el año 2011 se promulgó el Decreto Supremo no 054-PCM, con el que fue aprobado el llamado "Plan Bicentenario: el Perú hacia el 2021", formulado por el Centro de Planeamiento Nacional. Asimismo, el CEPLAN se propuso completar para el año 2050 un ambicioso plan en tres tiempos: el primer hito por cumplir el recién pasado año 2021 se ha conocido como Plan Bicentenario, el segundo hito ha sido establecido para el 2030 y el tercero para el 2050. Es importante señalar que las proyecciones fueron elaboradas en función del capital natural y la explotación de materias primas, por lo que la planificación no tiene claridad ni en la prospectiva ni en las estrategias, ya que la formulación del instrumento aparece como un fin y no como un medio capaz de lograr objetivos para el bienestar de los ciudadanos. Los planes estratégicos de desarrollo concertado llevan siendo formulados desde inicios del presente siglo por parte de los gobiernos regionales y locales, sin embargo, estos planes no han servido de guía para delinear las políticas públicas y son hasta ahora instrumentos de exiguo impacto debido a factores como el centralismo, los problemas políticos, los obstáculos jurídico-administrativos y la escasa dotación de recursos para la ejecución de los procesos.

En este periodo se aprobaron dos guías, una para la planificación estratégica y otra para la planificación territorial, ambas con serios problemas conceptuales y metodológicos. Entre las debilidades que presentan podemos mencionar: la escasa articulación de los instrumentos de planificación, la indefinición de los conceptos para ambos procesos, una metodología desfasada en relación con los cambios cada vez más dinámicos y la escasa multidisciplinariedad en la práctica planificadora. Considerando al auge del crecimiento económico y los problemas ambientales y sociales, durante la última década se han intensificado las actividades relacionadas con la planificación ambiental y estratégica. En 2012 se realizó el Primer Congreso Nacional de Planificación y Desarrollo Sostenible con el objetivo de evaluar los logros y las 
dificultades del proceso, evento al cual asistieron notables personalidades y representantes de las instituciones comprometidas en el proceso con el fin de evaluar sus avances y las debilidades. De las intervenciones en el evento se desprende que la planificación estratégica ha sido considerada como una herramienta fundamental para la búsqueda del bienestar y el desarrollo humano, y, tal como señaló Javier Iguiñiz en su condición de representante del Acuerdo Nacional, como un mecanismo adecuado para reducir las incertidumbres y disponer de planes de contingencia, prevención, mitigación y adaptación a situaciones adversas e inevitables (CEPLAN, 2012, p. 18).

Si bien la planificación estratégica es un proceso sistemático de ejecución de planes para lograr los objetivos planteados, es probable que funcione mejor en el ámbito privado que en el público, ya que la planificación estratégica demanda tiempo y requiere de capital humano e inversión (Hu et al., 2014), aspectos que no han sido considerados a la hora de elaborar los planes, cuyas metodologías tampoco han sido diseñadas adecuadamente para el proceso peruano. Además, los gobiernos de García y Humala tuvieron políticas de doble acción estatal, ya que por un lado promovieron la planificación y por el otro la obstaculizaron, como ocurrió con los procesos de zonificación ecológica económica orientados a los planes de ordenamiento territorial. Entre otros factores que intervinieron en esta doble acción estatal destacan la dispersión de competencias institucionales y el centralismo para la toma de decisiones estratégicas en torno a la planificación territorial peruana.

En la década pasada se redujeron las acciones de la planificación territorial y ambiental, dado que el CEPLAN promovió la planificación estratégica a través del Plan Estratégico Institucional (PEI), el Plan Operativo Institucional (POI) y los planes sectoriales. Si bien las acciones del CEPLAN se han orientado a fortalecer la planificación estratégica, esta no ha estado articulada con los planes territoriales y ambientales. Los lineamientos de la política para el ordenamiento territorial están a cargo del Ministerio del Ambiente y, según este organismo, el ordenamiento territorial se define como "un instrumento de planificación y gestión del desarrollo sostenible del país, para promover y regular los procesos de organización sostenible del territorio a nivel nacional, regional y local, articulados a los planes ambientales, de desarrollo, de desarrollo económico, social y otros" (Resolución Ministerial n 026, 2010). En definitiva, la planificación territorial como política estatal no ha sido delimitada de forma explícita a nivel competencial, conceptual y metodológico.

El último lustro se ha caracterizado por una inestabilidad permanente (2016-2021), puesto que en el Perú se han sucedido hasta cuatro presidentes de la república en un corto período de tiempo. En este contexto de conflictos políticos y corrupción, los temas relacionados con la planificación han quedado en un segundo plano. Las políticas emprendidas en los anteriores gobiernos se han discontinuado y la opinión del CEPLAN ha perdido peso en las decisiones políticas debido a que su metodología prospectiva no ha considerado el enfoque territorial en la concepción de los planes. Como ente rector en materia de planificación, actualmente el CEPLAN carece de autonomía y competencias, además de que cuenta con escasos recursos destinados a su gestión (Chiarella \& Yakabi, 2016). El problema de la planificación en Perú es la 'institucionalización y enfoque" (Fernández-Maldonado, 2019), así como la dispersión y el escaso compromiso político existente en algunos periodos gubernamentales. Su actual reto es superar la dispersión del cuerpo normativo y la burocracia administrativa, buscando un vínculo vertical y horizontal en la gestión de la información y el fortalecimiento de un ente con capacidad articuladora y decisión estratégica.

\section{Conclusión}

Como se ha visto a lo largo del estudio, la evolución de la planificación en Perú desde 1920 a 2021 se divide en cuatro períodos: incipiente (1920-1960), apogeo 
(1960-1980), crisis (1980-2000) y reinicio (2000-2021). No cabe duda de que las características de los cuatro períodos están intrínsecamente relacionadas con las directrices económicas de los diferentes gobiernos y con los acontecimientos políticos ocurridos en el país, aspectos que han determinado el éxito o el fracaso de la planificación en cada momento. Asimismo, del estudio se desprende que en el lapso que cubre los cuatro periodos el objetivo principal del planeamiento ha sido el desarrollo del país, por lo que es válido afirmar que el tipo de planificación preponderante en Perú, al margen de la existencia de las demás tipologías, ha sido la "planificación del desarrollo territorial", que ha buscado traducirse en el bienestar de los habitantes a escala nacional.

Del estudio también se desprende que la planificación peruana no ha tenido resultados homogéneos en los cuatro periodos. El momento más fructífero para la planificación regional fue durante el periodo de "apogeo", ya que la fundación del Instituto Nacional de Planificación le permitió contar con el apoyo estatal. Si bien destaca igualmente la decisión política del gobierno militar de Juan Velasco Alvarado de potenciarla, no alcanzó los resultados esperados debido a la crisis económica en el contexto internacional, ya que la crisis del petróleo acabó siendo determinante en la situación de los planes desarrollados en el Perú. Esta situación evidencia que los procesos de planificación de un país están supeditados a las políticas socioeconómicas y a las derivas políticas internas y externas que pueden influir en el éxito o el fracaso de los planes. Es así como tanto en el primero como en el tercer periodo los resultados fueron exiguos debido a circunstancias externas que no permitieron alcanzar los objetivos planteados en los planes.

En el cuarto periodo, la planificación estratégica, la territorial y la ambiental se han venido desarrollando de forma desarticulada. Como institución responsable de la planificación nacional, el Centro Nacional de Planeamiento Estratégico (CEPLAN) no ha podido consolidarse como una instancia articuladora y motora del proceso de planificación. Esto nos lleva a concluir que existen dudas razonables respecto al futuro del proceso de planificación en el Perú, ya que, por un lado, hay una gran necesidad de contar con planes, pero, por el otro, existe un poderoso sector reticente que ve en la planificación un medio de control por parte del Estado. Finalmente, como sugerencia conclusiva, es relevante señalar que, por su carácter holístico, la planificación territorial debe implementarse en instancias regionales, articulándose verticalmente a nivel nacional y local, y de forma horizontal con los sectores y los organismos que operan en los ámbitos regionales.

\section{Referencias}

Bielza de Ory, V. (2002). Experiencias y replanteamientos de la ordenación del territorio en Europa: retos en relación con la ciudad. Geographicalia, $41,37-57$

Bielza de Ory, V. (2012). Organización y ordenamiento del territorio. Desarrollo y sostenibilidad. Experiencia europea para el Perú. Revista Geográfica, 152, 83-103.

Bielza de Ory, V. \& Bohl, R. (2012). Ordenación territorial y turismo en Latinoamérica: el caso peruano. Revista Geográfica, 151, 63-81.

Capel, H. (2016). Las ciencias sociales y el estudio del territorio. Biblio3W. Revista Bibliográfica de Geografia y Ciencias Sociales, 21(1149). http://www. ub.edu/geocrit/b3w-1149.pdf

Castillo, R. (2021). Evolución de la planificación urbana en el Perú 1946-2021: de la planificación urbana normativa a la planificación del desarrollo urbano sostenible. Paideia, 11(1), 79-112. https://revistas.urp.edu.pe/index.php/Paideia/ article/view/3783

Centro Nacional de Planeamiento Estratégico (CEPLAN) (2012). $1^{\circ}$ Congreso Nacional de Planificación y Desarrollo Sostenible: crecimiento con inclusión y sostenibilidad ambiental. Cusco, Perú.

Centro Nacional de Planeamiento Estratégico (CEPLAN) (2015). Visión estratégica del planeamiento. El PEDN al 2021. https://bioseguridad.minam. gob.pe/wp-content/uploads/2016/09/ceplan_ moraotria.pdf 
Chiarella, J. \& Yakabi, K. (2016). Planificación y ordenamiento territorial. Consideraciones a partir del caso peruano. Revista Política e Planeamiento Regional, 3(2), 137-158.

Comisión Económica para América Latina y el Caribe (CEPAL), Instituto Latinoamericano y del Caribe de Planificación Económica y Social (ILPes) (1978). Información básica sobre la organización y funcionamiento del Sistema Nacional de Planificación del Perú. Boletín de Planificación, 4-5, 97-108.

Comisión Económica para América Latina y el Caribe (CEPAL) (1986). Desarrollo social y pobreza en Perú. Factores estructurales y efectos de la crisis externa. Las politicas adoptadas para lograr el desarrollo económico y social. CEPAL.

Conferencia Europea de Ministros Responsables de Ordenación del Territorio (СЕмAT) (1983). Carta Europea de Ordenación del Territorio.

Constitución Política del Perú (1979), publicada el 12 de julio.

Constitución Política del Perú (1993), publicada el 30 de diciembre.

Coraggio, J. (1981). Las bases teóricas de la planificación regional en América Latina. En S. Boisier, F. Cepeda, J. Hilhorst, S. Riffka \& F. Uribe-Echevarría (eds.), Experiencias de planificación regional en América Latina: una teoría en busca de una práctica (pp. 147-180). ILPES.

Decreto Legislativo n 177 (1981, junio 12), Ley Orgánica del Sistema Nacional de Planificación. El Peruano, 13 de junio. http://portal.apci.gob. pe/Compendio\%20Normas\%20Legales $\% 20$ СTI/003.pdf

De Lisio, A. (2014). Brasil en el camino del ordenamiento territorial "glocal". Terra Nueva Etapa, 30(48), 61-87.

Dourojeanni, M. (2016). ¿Existe planificación en el Perú? http://www.actualidadambiental.pe/?P=34799

Elizalde, A. (2003). Planificación estratégica territorial y políticas públicas para el desarrollo local. CEPAL.

Fernández-Maldonado, A. (2019). Unboxing the Black Box of Peruvian Planning. Planning Practice E Research, 34(4), 368-386. https://doi.org/10.1 080/02697459.2019.1618596

Fitzgerald, E. (1981). La economía política del Perú 19561978: desarrollo económico y reestructuración del capital. Instituto de Estudios Peruanos.
Galilea, S. (1979). La planificación regional en los países pequeños: referencias preliminares a la estrategia de necesidades básicas. Revista Eure, 21, 17-32.

Gallardo, R. (2013). Rompiendo paradigmas: la planificación del siglo XXI. https://www.oas.org/es/sap/ dgpe /docs $/ \mathrm{la} \% 20$ planificacion $\% 20 \mathrm{del} \% 20$ siglo $\% 20 x x i \% 20$ (final).pdf

Glave, M. (2012). Ordenamiento territorial y desarrollo en el Perú: notas conceptuales y balance de logros y limitaciones. En Desarrollo rural y recursos naturales (pp. 123-165). GRADE; Grupo Propuesta Ciudadana.

Guimaraes, R. (2001). Fundamentos territoriales y biorregionales de la planificación. CEPAL.

Hilhorst, J. (1979). Planificación y desarrollo regional en el Perú: 1968-1977. EURE, Revista de Estudios Urbano Regionales, 6(17), 23-41.

Hu, Q., Kapucu, N., \& O’Byrne, L. (2014). Strategic Planning for Community-Based Small Nonprofit Organizations: Implementation, Benefits, and Challenges. The Fournal of Applied Management and Entrepreneurship, 19(1), 83-101. https://www.academia.edu/5940902/Strategic_planning_and_ management_for_small_nonprofit_organizations_Perception_implementation_and_impact

Lajo, M. (1980). Del Plan Inca al Plan Túpac Amaru. Comercio Exterior, 28(2), 197-205.

Leceta, H. (2012). Historia de la planificación en el Perú en el cincuentenario de su institucionalización. Centro Nacional de Planeamiento Estratégico (CEPLAN).

Leyton, G. \& López, J. (2005). Plan de desarrollo concertado y presupuesto participativo 2004-2005. Avances y desafios a tomar en cuenta. Cuadernos Descentralistas, $\mathrm{n}^{\circ} 14$.

Lira, L. (2006). Revalorización de la planificación del desarrollo. Instituto Latinoamericano y del Caribe de Planificación Económica y Social (ILPES).

Mata, A. \& Quevedo, F. (1994). Diccionario didáctico de ecología. Editorial de la Universidad de Costa Rica.

Moschella, P. (2012). Variación y protección de humedales costeros frente a procesos de urbanización: casos Ventanilla y Puerto Viejo. [Tesis de Grado]. Pontificia Universidad Católica del Perú.

Pemán, I. (2011). Glosario sobre desarrollo territorial. Conferencia europea de ministros responsables en materia de ordenación del territorio (СЕMAT). Revista Aragonesa de Administración Pública, 38, 467-504. 
Ponce, L. (2014). Política fiscal del Perú, 1920-1980. En C. Contreras (ed.), Compendio de historia económica del Perú. (Tomo 5. La economía peruana entre la gran depresión y el reformismo militar, 1930-1980, pp. 345-414). Instituto de Estudios Peruanos (IeP); Banco Central de Reserva del Perú (BCRP).

Portocarrero, F. (1989). La economía peruana en los años 80. Apuntes, 25, 105-118.

Resolución Ministerial n 026-2010-MINAM (2010, febrero 26). Aprobación de los Lineamientos de Política para el Ordenamiento Territorial. https://www. minam.gob.pe/wp-content/uploads/2013/09/ rm-026-2010-minam.pdf

Rendón, S. (2019). Ordenamiento y planificación territorial en Perú. Una aproximación crítica a los instrumentos de Zonificación Ecológica Económica (ZEE). [Tesis de doctorado]. Universidad de Barcelona. http://hdl. handle.net/2445/146803

Reyna, C. (2000). La anunciación de Fujimori: Alan García 1985-1990. Centro de Estudios y Promoción del Desarrollo (DESCO).
Sánchez, F. (2003). Constitución política, acuerdo nacional y planeamiento estratégico en el Perú. (Serie Gestión Pública). Instituto Latinoamericano de Planificación Económica y Social (ILPES).

Sánchez, J. (2002). La revolución peruana: ideología y práctica politica de un gobierno militar 1968-1975. Consejo Superior de Investigaciones Científicas (CSIC).

Servillo, L. \& Van Den Broeck, P. (2012). The Social Construction of Planning Systems: A Strategic-Relational Institutionalist Approach. Planning Practice and Research, 27(1), 41-61. https://doi.org /10.1080/02697459.2012.661179

Velasco, O. (2013). Perú. La dificil construcción de una república para todos. Instituto de Gobierno; Universidad de San Martín de Porras.

Zegarra, L. (2020). Perú, 1920-1980. Contexto internacional, políticas públicas y crecimiento económico. En C. Contreras (ed.), Compendio de historia económica del Perú. (Tomo 5. La economía peruana entre la gran depresión y el reformismo militar, 1930-1980, pp. 19-104). Instituto de Estudios Peruanos (IEP); Banco Central de Reserva del Perú (BCRP). https://www.bcrp.gob.pe/docs/Publicaciones/libros/historia/economia/5-gran-depresion-y-reformismo-militar.pdf 